\title{
Eficiencia en la reducción de Cromo por una bacteria silvestre en un tratamiento tipo Batch utilizando como sustrato agua residual del municipio de Pasto, Colombia
}

\author{
Efficiency in the reduction of chromium by a wild bacterium in a Batch treatment type using residual water \\ substrate from the municipality of Pasto, Colombia
}

\author{
Deisy Lorena Guerrero Ceballos ${ }^{*}$ orcid.org/0000-0001-8960-8538 \\ Jhonatan Pinta-Melo' ${ }^{1}$ orcid.org/0000-0003-0347-2559 \\ Pablo Fernández-Izquierdo1,2 orcid.org/0000-0003-0158-8398 \\ Eduardo Ibargüen-Mondragón ${ }^{2,3}$ orcid.org/0000-0001-6308-1344 \\ Sandra Patricia Hidalgo-Bonilla 4 orcid.org/0000-0001-8905-8716 \\ Edith Mariela Burbano-Rosero',2 orcid.org/0000-0002-4021-2660
}

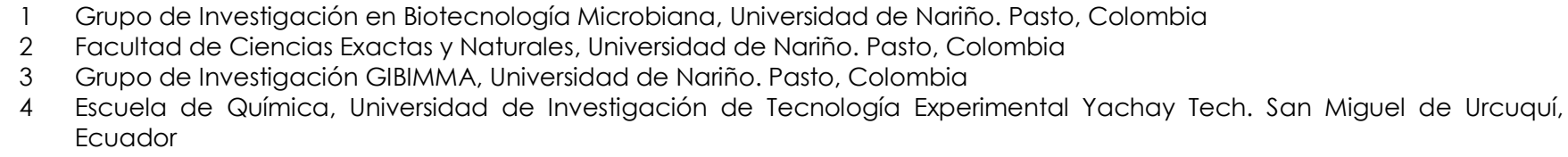

\section{Resumen}

Objetivo: Evaluar la eficiencia en la reducción de Cromo en un tratamiento tipo Batch, utilizando como sustrato agua residual municipal inoculada con una bacteria silvestre. Materiales y métodos: Se verificó a escala de laboratorio el porcentaje de reducción de Cromo hexavalente de tres bacterias silvestres previamente aisladas de agua residual del Río Pasto (Bacillus thuringiensis, Bacillus amyloliquefaciens y Paenibacillus sp.); se seleccionó el aislado que presentó mayor porcentaje de reducción de $\mathrm{Cr}$ y fue sometido a diferentes tratamientos. El análisis de los resultados se hizo mediante estadística descriptiva. Resultados: B. thuringiensis, B. amyloliquefaciens, y Paenibacillus sp., presentaron porcentajes de reducción de $\mathrm{Cr}$ (VI) de 82,01\%; 80,85\% y 79,27\%, respectivamente. Se determinó que el tercer tratamiento (agua sin esterilizar del Río Pasto con B. thuringiensis) presentó diferencias significativas respecto a los demás ( $\mathrm{p}=0,0001 \alpha=0,05)$, concluyendo que B. thuringiensis reduce en mayor proporción el $\mathrm{Cr}(\mathrm{VI})$, los resultados encontrados en esta investigación son promisorios en el campo de la biorremediación de efluentes contaminados con Cromo ya que pueden ser tomados como base para implementar estrategias de biorremediación a gran escala. Conclusión: La bacteria B. thuringiensis presentó alta eficiencia en la reducción de Cromo hexavalente $(99,42 \%)$, cuando fue implementada en un tratamiento a escala de laboratorio de agua residual sin esterilizar.

Palabras clave: Metales pesados; remoción de contaminantes, biorremediación. (Fuente: DeCS, Bireme).

\begin{abstract}
Objective: To evaluate the efficiency in the reduction of chromium in a Batch treatment type, using municipal residual water substrate inoculated with a wild bacterium. Materials and methods: The reduction percentage of
\end{abstract}


hexavalent chromium of three wild bacteria previously isolated from residual water from the Pasto River was verified at laboratory scale (Bacillus thuringiensis, Bacillus amyloliquefaciens and Paenibacillus $s p$.); the isolated that showed the highest percentage of reduction of $\mathrm{Cr}$ was selected and was subjected to different treatments. The analysis of results was done using descriptive statistics. Results: B. thuringiensis, B. amyloliquefaciens, and Paenibacillus sp., presented percentages of reduction of $\mathrm{Cr}(\mathrm{VI})$ of 82,01\%; 80,85\% and 79,27\%, respectively. It was determined that the third treatment (nonsterile water from the Pasto River with B. thuringiensis) presented significant differences with regard to the other ( $p=0.0001 \alpha=0.05)$, concluding that $B$. thuringiensis reduces in greater proportion the $\mathrm{Cr}(\mathrm{VI})$. The results found in this research are promising in the field of bioremediation of contaminated effluents with Chrome since they may be taken as the basis for implementing strategies of bioremediation on a large scale. Conclusion: The bacteria B. thuringiensis presented high efficiency in the reduction of hexavalent chromium (99.42\%) when implemented in a treatment at laboratory scale of residual nonsterile water.

Keywords: Heavy metals; reduction of chromium; wild bacteria; reducing bacteria Cr. (Source: DeCS, Bireme).

\section{Introducción}

El Cromo (Cr) es uno de los metales pesados (MP) de mayor impacto ambiental por encontrarse en altas concentraciones en la mayoría de desechos generados en actividades industriales ${ }^{1}$, principalmente: galvanizado, aleaciones, procesos de curtido y tinción de textiles. Los efectos del Cromo en el ambiente dependen del estado de oxidación, entre los más estables está la forma trivalente, Cr(III), y hexavalente, $\operatorname{Cr}(\mathrm{VI})$, siendo esta última la más tóxica por su alta solubilidad en agua, alto grado de oxidación y capacidad para permear membranas biológicas, ocasionando alteraciones en el genoma ${ }^{2,3}$.

Actualmente, la Agencia de Protección Ambiental de Estados Unidos (EPA), ha establecido un nivel de contaminación máximo de $0.1 \mathrm{mg} / \mathrm{L}$ para la cantidad total de Cromo en el agua potable. Debido a la peligrosidad que presenta el Cromo hexavalente, el Departamento de Salud y Servicios Humanos (DHHS), la Agencia Internacional para la Investigación del Cáncer (IARC) y la EPA han determinado que los compuestos de Cromo (VI) son carcinogénicos en seres humanos 4 .

En el departamento de Nariño las sales de Cromo son ampliamente usadas en la industria del curtido, sus residuos son vertidos directa e indirectamente a las principales fuentes hídricas, ocasionando un desequilibrio ecológico y biogeoquímico. Estudios previos realizados por Arango y Alzate $^{5}$ determinaron una concentración de $59 \mathrm{mg} / \mathrm{L}$ de Cr (VI) en los puntos de vertimiento en el sector de Pandiaco, donde se localizan las curtiembres. En este sentido, se han considerado varias alternativas para la recuperación de este tipo de efluentes, entre las que sobresalen: la implementación de métodos fisicoquímicos, síntesis de arcillas aniónicas y fotocatálisis. No obstante, estos procesos son limitados por el alto costo económico, elevado consumo de energía y la emisión de otro tipo de contaminantes ${ }^{6-10}$.

Desde esta perspectiva, en Nariño se evaluó en un estudio preliminar la producción de polihidroxialcanoatos a partir de bacterias provenientes de muestras de agua del Río Pasto, como una estrategia para disminuir la carga orgánica acumulada en las aguas residuales vertidas al río; a partir de este se obtuvo un banco de aislados bacterianos con posibles aplicaciones biotecnológicas ${ }^{11}$. Posteriormente, se evaluó la capacidad reductora de $\operatorname{Cr}(\mathrm{VI})$ del banco de aislados en procesos de fermentación en medios de cultivo sintéticos, con base en este ensayo se seleccionaron los aislados que presentaron un rápido crecimiento en medios suplementados con Cromo hexavalente ${ }^{12}$.

Las bacterias fueron identificadas mediante técnicas moleculares basadas en la amplificación y secuenciación del gen $16 \mathrm{~S}$ rRNA, dicho estudio identificó que estas pertenecían a las especies Bacillus thuringiensis, Bacillus amyloliquefaciens y Paenibacillus sp., complementariamente se determinó la tolerancia de estos tres aislados bacterianos en concentraciones de Cr (VI) a 25, 
60 y $100 \mathrm{mg} / \mathrm{L}^{13}$. Sin embargo, no existe información acerca de la eficiencia en la reducción de Cromo que podrían presentar estas bacterias en agua residual, por esto se evaluó la eficiencia en la reducción de $\mathrm{Cr}$ (VI) de los aislados bacterianos al ser sometidos a procesos de fermentación en los que se implementó como sustrato agua del Río Pasto contaminado con este metal, en un tratamiento tipo Batch ${ }^{14,15}$. Los resultados encontrados, respecto al porcentaje de reducción de Cromo por una bacteria silvestre, abren importantes perspectivas para implementar estrategias que permitan la biorremediación de efluentes contaminados.

\section{Materiales y métodos}

\section{Viabilización de aislados bacterianos}

Los aislados bacterianos fuente de este estudio $B$. amyloliquefaciens, $B$. thuringiensis y Paenibacillus $s p$., fueron proporcionados por el grupo de Biotecnología Microbiana de la Universidad de Nariño ${ }^{13}$, se conservaron en agar inclinado (agar nutriente) con glicerol al $30 \%$ y fueron refrigerados a $-4{ }^{\circ} \mathrm{C}$ usando un Freezer Isotemp marca Fisher Scientific por aproximadamente 24 meses antes de la realización del presente estudio. Los aislados se viabilizaron en tubos de ensayo con caldo Luria Bertani (LB), luego de $18 \mathrm{~h}$ de incubación a $30{ }^{\circ} \mathrm{C}$, se verificó su condición axénica mediante tinción de Gram; posteriormente se realizó el proceso de crioconservación, para lo cual se inocularon 100 $\mu \mathrm{L}$ de cada aislado (previamente crecido), en crioviales con $900 \mu \mathrm{L}$ de caldo LB-glicerol 30\%, posteriormente, se llevaron a $-20{ }^{\circ} \mathrm{C}$. Para cada aislado se conservaron 10 criovales.

\section{Descripción del sitio y características de la muestra}

La ciudad de San Juan de Pasto posee varios puntos de vertimiento al Río Pasto, uno de ellos es el colector Juan 23 ubicado en el sector Torobajo donde se obtuvieron las muestras de agua residuales objeto de esta investigación

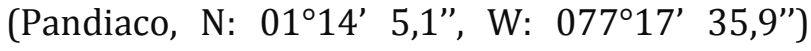
entre el mes de octubre y noviembre de 2015 (Figura 1). El agua se transportó en recipientes plásticos de $20 \mathrm{~L}$, fue filtrada previo a su uso con algodón, gasa y un tamiz $\mathrm{N}^{\circ} 200$ para evitar el ingreso de partículas gruesas. La muestra se diluyó en agua tamponada a pH 7,0 (Solución Salina Amortiguada por Fosfatos, PBS) en una proporción 50:50, esterilizada previamente y suplementada con $59 \mathrm{mg} / \mathrm{L}$ de dicromato de potasio. La esterilización del agua se realizó usando una autoclave a $121{ }^{\circ} \mathrm{C}$ durante 15 minutos, procedimiento que se repitió por duplicado cada 24 horas.

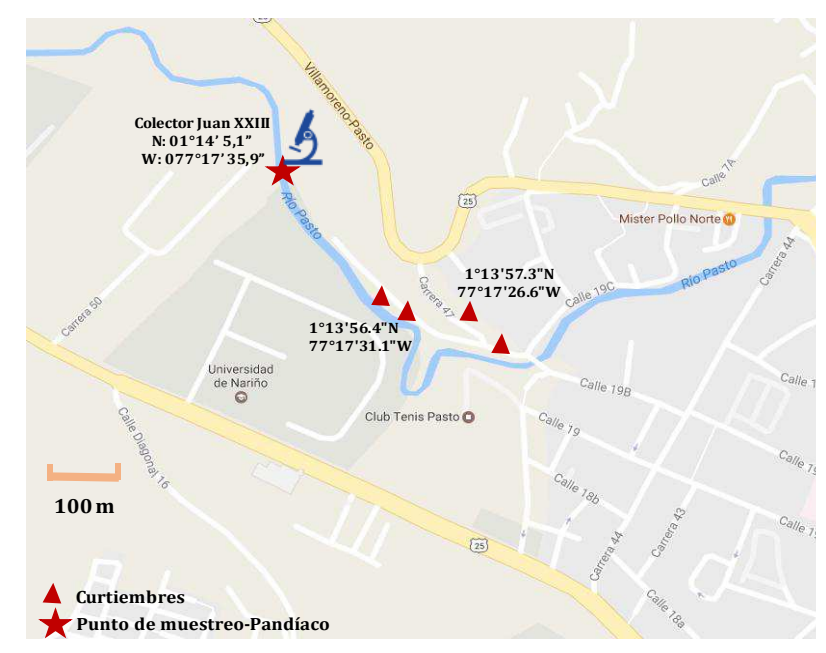

Fuente: El mapa fue construido usando el programa de libre acceso: https://www.openstreetmap.org/node/309303769\#map=15/1.22 46/-77.2786

Figura 1. Mapa del punto de vertimiento y de muestreo, colector Juan 23

\section{Diseño Experimental}

Como unidad experimental se emplearon biorreactores de $60 \mathrm{~cm}$ de largo por 11,4 cm de diámetro. En la primera fase de la investigación se realizaron dos réplicas biológicas de cada bacteria (B. amyloliquefaciens, B. thuringiensis y Paenibacillus sp.), las cuales presentaban a su vez tres réplicas técnicas; los biorreactores se operaron por $156 \mathrm{~h}$, tiempo en el cual se tomaron muestras de $5 \mathrm{~mL}$ cada 12 horas para la determinación de Cromo hexavalente. En la segunda fase de la investigación se trabajó con el modelo seleccionado en la fase anterior y se realizaron tres replicas biológicas de cada tratamiento (agua sin esterilizar del Río Pasto; agua estéril del Río Pasto inoculada con $B$. thuringiensis y agua sin esterilizar del Río Pasto inoculada con $B$. thuringiensis), con tres replicas técnicas cada uno; de igual manera, los 
biorreactores se operaron por $156 \mathrm{~h}$, tiempo en el cual se tomaron muestras de $5 \mathrm{~mL}$ cada 12 horas para la determinación de Cromo hexavalente (Figura 2).
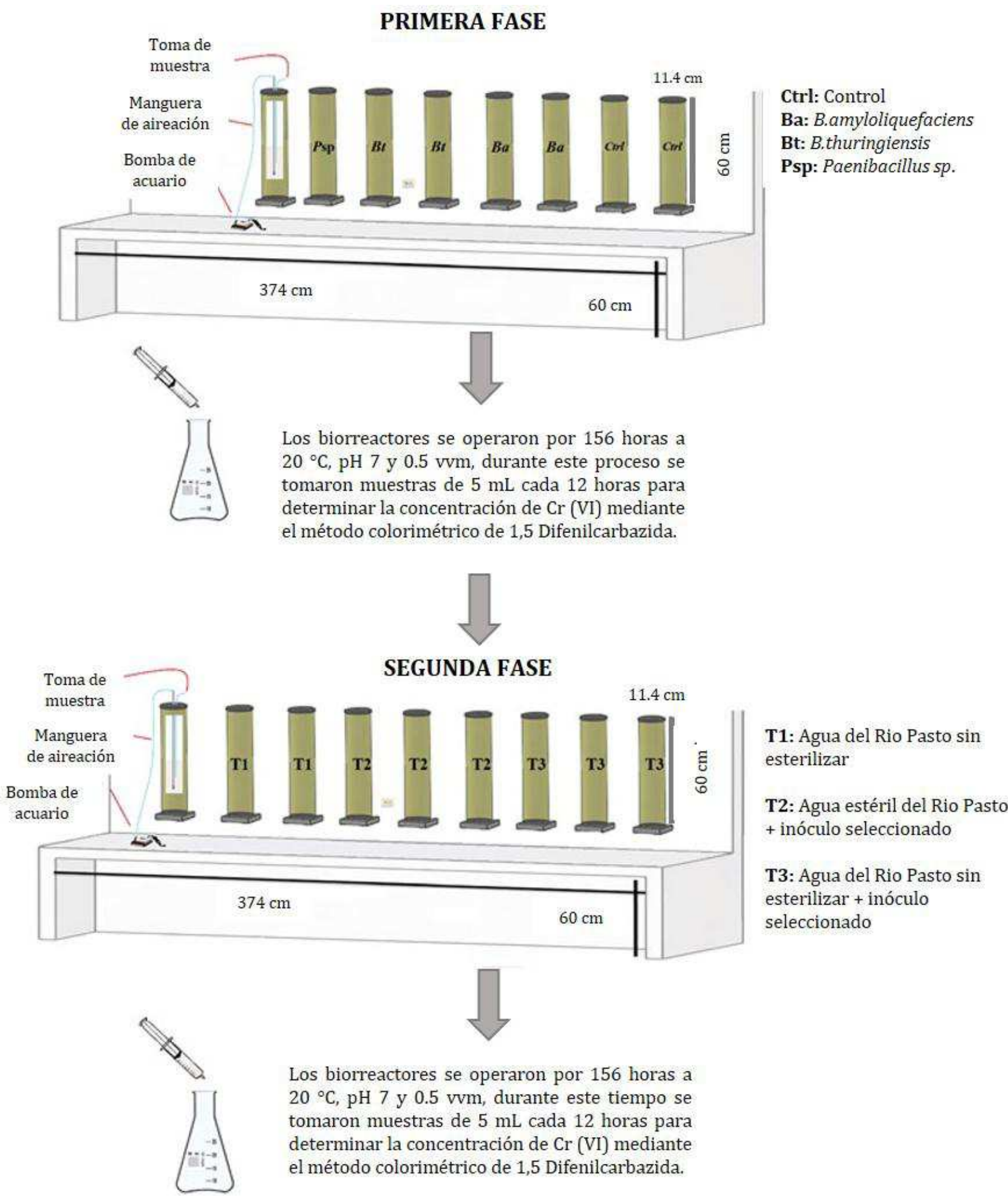

Los biorreactores se operaron por 156 horas a $20^{\circ} \mathrm{C}$, pH 7 y $0.5 \mathrm{vvm}$, durante este proceso se tomaron muestras de $5 \mathrm{~mL}$ cada 12 horas para determinar la concentración de $\mathrm{Cr}$ (VI) mediante el método colorimétrico de 1,5 Difenilcarbazida.

Fuente: La figura fue diseñada usando el Software GNU image manipulation program con licencia pública: http://www.gnu.org/licenses/

Figura 2. Diseño experimental (A) réplicas primera fase y (B) réplicas segunda fase 


\section{Cuantificación de Cromo hexavalente}

Para cuantificar la reducción de Cromo de los aislados bacterianos en las muestras se realizó una curva patrón, para ello se analizaron químicamente cinco soluciones patrón usando el método de la 1,5-difenilcarbazida, el cual contempla que a cada solución se le adicione 2 gotas de ácido sulfúrico $\left(\mathrm{H}_{2} \mathrm{SO}_{4}\right)$ al $20 \%, 2$ gotas ácido fosfórico $\left(\mathrm{H}_{3} \mathrm{PO}_{4}\right)$ al $80 \%$ y $2 \mathrm{~mL}$ de solución de 1,5-difenilcarbazida $\left(\left(\left(\mathrm{C}_{6} \mathrm{H}_{5}\right) \mathrm{NHNH}\right)_{2} \mathrm{CO}\right)$ al $85 \%$ disuelta en acetona. La absorbancia de todas las soluciones patrones fue tomada a una longitud de onda de $543 \mathrm{~nm}$, se usaron celdas de vidrio de paso óptico de $1 \mathrm{~cm}$ y posteriormente se analizó la correlación por mínimos cuadrados entre la absorbancia y la concentración usando el programa estadístico Statgraphics Centurión XVI de libre acceso. La solución patrón más concentrada, $100 \mathrm{~mL}$, fue preparada usando $1 \mathrm{~mL}$ de una solución madre de Cromo (VI) $(0,142 \mathrm{~g}$ de dicromato de potasio anhidro en $100 \mathrm{~mL}$ de agua desionizada) у a partir de esta solución patrón se realizaron cinco diluciones patrón de $25 \mathrm{~mL}$ de concentraciones entre 0,2 y $1 \mathrm{mg} / \mathrm{L}$ de $\mathrm{Cr}(\mathrm{VI})$. El análisis químico de las cinco soluciones patrón fue llevado a cabo usando el método de la 1,5- difenilcarbazida, adicionando $\mathrm{H}_{2} \mathrm{SO}_{4}, \mathrm{H}_{3} \mathrm{PO}_{4}$ y 1,5 difenilcarbazida se homogenizó, se dejó reposar hasta obtener el desarrollo del color característico del complejo formado y se determinó la absorbancia de todas las soluciones patrones ${ }^{16}$.

\section{Selección de la bacteria con mayor eficiencia en la reducción de Cromo hexavalente en agua residual municipal estéril}

Para garantizar la densidad poblacional estándar de las bacterias inoculadas (B. amyloliquefaciens, $B$. thuringiensis y Paenibacillus sp.) al iniciar el proceso de verificación del porcentaje de reducción de $\mathrm{Cr}(\mathrm{VI})$, se trabajó a partir de un inóculo bacteriano de $10^{8}$ bacterias por mililitro (escala McFarland, tubo 5) a una longitud de onda de $540 \mathrm{~nm}$ el cual fue verificado por la medición de turbidez. El tratamiento control constó de 3,5 L de agua residual municipal estéril. Se usaron biorreactores tipo Batch, los cuales se operaron por $156 \mathrm{~h}$ a $20^{\circ} \mathrm{C}, \mathrm{pH} 7,0$ y un flujo de aire de $0,5 \mathrm{vvm}$ (volumen de aire por volumen de medio por minuto) ${ }^{17}$; la concentración de Cr (VI) en la muestra de agua se logró a través la adición de Cromo sólido en forma de dicromato de potasio, $59 \mathrm{mg} / \mathrm{L}$.

Las muestras de agua del colector Juan 23, fueron analizadas usando el método de la 1,5difenilcarbazida, a cada alícuota de $5 \mathrm{~mL}$ de muestra diluida (factor de dilución 1/100) y tomada por triplicado cada $12 \mathrm{~h}$, se adicionó $\mathrm{H}_{2} \mathrm{SO}_{4}, \quad \mathrm{H}_{3} \mathrm{PO}_{4}$ y 1,5 difenilcarbazida, se homogenizó y se dejó reposar hasta obtener el desarrollo del color rojo violeta característico de la formación del complejo; consecutivamente, se determinó la concentración de Cr (VI) y el porcentaje de reducción, usando la ecuación de la recta obtenida de la curva de calibración y la ecuación 118, respectivamente. Teniendo en cuenta los resultados obtenidos en esta fase, se graficó la cinética de reducción de las tres bacterias y se seleccionó el aislado bacteriano que presentó mayor porcentaje de reducción de Cr (VI) ${ }^{16}$.

$$
\begin{array}{r}
\text { \% reducción }[\operatorname{Cr}(V I)]= \\
\frac{[\operatorname{Cr}(V I)]_{\text {inicial }}-[\operatorname{Cr}(V I)]_{\text {final }}}{[\operatorname{Cr}(V I)]_{\text {inicial }}} * 100
\end{array}
$$

\section{Evaluación de la eficiencia en la reducción de Cr (VI) de Bacillus thuringiensis en un tratamiento tipo Batch utilizando como sustrato agua residual municipal}

Del análisis de reducción de Cromo hexavalente por los tres aislados bacterianos se seleccionó un aislado bacteriano el cual fue sometido a un proceso de fermentación cerrado, empleando biorreactores tipo Bach con una capacidad total de $5 \mathrm{~L}$ y volumen efectivo de 3,5 L; como sustrato se utilizó agua sin esterilizar del Río Pasto, diluida en agua tamponada a pH 7,0 en una proporción 50:50 y suplementada con $59 \mathrm{mg} / \mathrm{L}$ de dicromato de potasio. Se trabajó a partir de un inóculo al $10 \%$ del aislado bacteriano, el cual se viabilizó en caldo LB y se inoculó en el biorreactor manteniendo constante las condiciones de temperatura, $\mathrm{pH}$ y vvm, durante 156 h; el tratamiento constó de dos controles, el primero de 3,5 L de agua sin esterilizar del Río Pasto y el segundo 3,5 L de agua estéril del Río Pasto y el inóculo bacteriano. 


\section{Análisis de los datos}

Los análisis estadísticos de los datos obtenidos para las muestras de río Pasto fueron evaluados mediante test ANOVA, análisis de varianza y gráficos de correlación, usando el programa Statgraphics Centurión XVI. Para el análisis de la reducción de Cromo por $B$. amyloliquefaciens, $B$. thuringiensis y Paenibacillus sp., se usó varianza simple (ANOVA) y se plantearon la hipótesis nula (todos los tratamientos son iguales) y la hipótesis alternativa (al menos uno de los tratamientos es distinto a los demás) con el fin de determinar si existen diferencias significativas en el porcentaje de reducción de $\mathrm{Cr}$ (VI) realizado por $B$. amyloliquefaciens, $B$. thuringiensis y Paenibacillus sp. De igual forma, para el análisis de los resultados obtenidos en la evaluación de la eficiencia en la reducción de $\mathrm{Cr}$ (VI) por el aislado seleccionado, se realizó un análisis de varianza (ANOVA) con el fin de determinar si existen diferencias significativas en la eficiencia de reducción de $\mathrm{Cr}$ (VI) del tratamiento con el inóculo seleccionado, respecto a los controles. Posteriormente, se realizó la prueba de Tukey para verificar en que tratamientos se presentan diferencias significativas.

\section{Consideraciones éticas}

Esta investigación fue catalogada según el artículo 11 de la Resolución 8430 de 4 de octubre de 1993 del Ministerio de Salud, como de riesgo mínimo. Los microorganismos manejados en el estudio corresponden a tipo de riesgo I o II, que con base en el artículo 68 de la Resolución deben manejarse en laboratorios de tipo básico de microbiología, empleando gabinetes de seguridad, cuando se considere necesario.

\section{Resultados}

\section{Cuantificación de Cromo hexavalente}

La cuantificación de Cromo hexavalente por análisis colorimétrico de la 1,5-difenilcarbazida, es un método específico para Cromo (VI) que proporciona resultados exactos ${ }^{19,20,21}$.
La Figura 3 relaciona la concentración de las cinco soluciones patrón en función de la absorbancia medida a una longitud de onda de $543 \mathrm{~nm}$, dichas mediciones fueron realizadas por triplicado (Tabla 1) y se fijó como variable dependiente la absorbancia y como variable independiente la concentración de $\mathrm{Cr}$ (VI) $(A=$ $0,0073+0,7005^{*}[\mathrm{Cr}]$ ) obteniendo un coeficiente de correlación de 0,999 , mostrando la linealidad predicha por la ley de Lambert-Beer ${ }^{22}$.

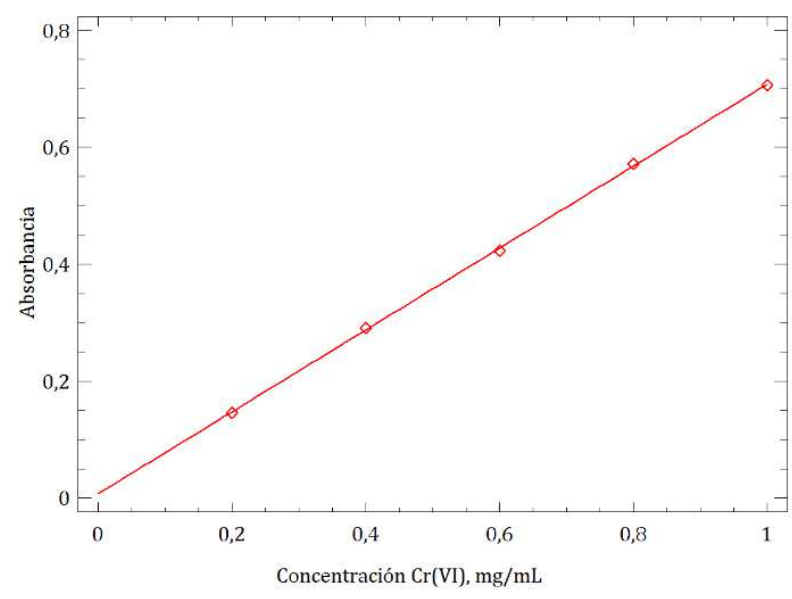

Figura 3. Curva de calibración (Concentración vs absorbancia) por análisis colorimétrico

Con el fin de verificar la precisión y confiabilidad de los datos de absorbancia, se determinó el coeficiente de variación (CV) de cada dato, como medida estadística de las dispersiones de los resultados, y así proporcionar una aproximación de la calidad de los datos. Teniendo en cuenta que a mayor valor de CV mayor heterogeneidad entre los datos de absorbancia y a menor CV, mayor homogeneidad.

Relacionando los valores del CV se determinó que los datos presentan buena reproducibilidad de los resultados, los valores de absorbancia resultaron en su totalidad ser homogéneos para cada concentración (Tabla 1). Este resultado es respaldado al analizar la desviación estándar, $\sigma$, mostrando que existe una mayor concentración de datos alrededor de la media. 
Tabla 1. Datos usados en la curva de calibración y datos para análisis estadístico

\begin{tabular}{|c|c|c|c|c|c|c|}
\hline \multirow{2}{*}{$\begin{array}{l}\text { Concentración } \\
\text { Cr (VI) mg/L }\end{array}$} & \multicolumn{3}{|c|}{ Absorbancia a $543 \mathrm{~nm}$} & \multirow{2}{*}{$\begin{array}{l}\text { Promedio } \\
\text { absorbancia }\end{array}$} & \multirow[b]{2}{*}{$\sigma$} & \multirow{2}{*}{ CV \% } \\
\hline & Muestra 1 & Muestra 2 & Muestra 3 & & & \\
\hline 0,2 & 0,152 & 0,146 & 0,141 & 0,146 & 0,006 & $3,76 \%$ \\
\hline 0,4 & 0,283 & 0,292 & 0,297 & 0,291 & 0,007 & $2,44 \%$ \\
\hline 0,6 & 0,423 & 0,430 & 0,416 & 0,423 & 0,007 & $1,65 \%$ \\
\hline 0,8 & 0,569 & 0,596 & 0,550 & 0,572 & 0,023 & $4,04 \%$ \\
\hline 1,0 & 0,709 & 0,704 & 0,706 & 0,706 & 0,003 & $0,36 \%$ \\
\hline
\end{tabular}

Al realizar un análisis de la varianza por ANOVA simple, se concluye que existe una relación estadísticamente significativa entre media de la absorbancia entre un nivel de concentración de $\mathrm{Cr}$ (VI) y otro dado que el valor de p es menor que $0,05(p=0)$, con un nivel de confianza del $95,0 \%$.

\section{Selección de la bacteria con mayor eficiencia en la reducción de Cromo hexavalente en agua residual municipal estéril}

Una vez finalizado el proceso de fermentación, se determinó el porcentaje de reducción de Cromo (VI) final realizado por cada aislado bacteriano, el cual fue de $83,05 \%, 80,95 \%$ y $79,52 \%$ para $B$. thuringiensis, $\quad$ B. amyloliquefaciens, $\quad \mathrm{y}$ Paenibacillus $s p$, respectivamente. Se utilizaron los datos de la variación de la concentración de Cromo hexavalente con respecto al tiempo para analizar la cinética de reducción de este metal para cada bacteria y el control (Figura 4).

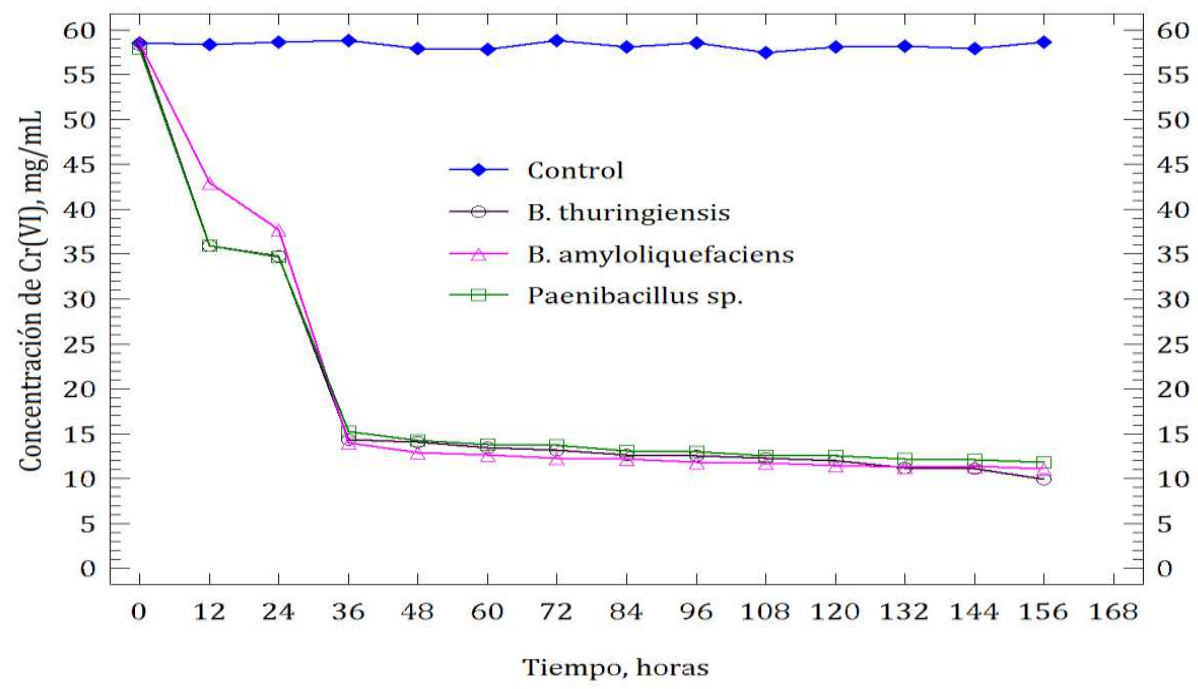

Figura 4. Cinética de la reducción de Cromo hexavalente de Bacillus thuringiensis, Bacillus amyloliquefaciens, y Paenibacillus sp. en agua residual municipal estéril

En la figura 4 se muestra que existen, en general, dos comportamientos en la reducción de la concentración de Cromo (VI) en los intervalos de tiempo de 0 - 36 horas y 36 - 156 horas. Una fase inicial rápida ( 0 - 36 horas), para los tres aislados bacterianos, donde los porcentajes de reducción de $\mathrm{Cr}$ (VI) fueron de 76,15\%; 75,46\% y $73,60 \%$ para B. thuringiensis, $B$. amyloliquefaciens, y Paenibacillus sp, respectivamente. Aplicando mínimos cuadrados, 
a esta fase, los datos de concentración de Cromo hexavalente y tiempo para cada aislado se puede proponer que la cinética de reducción es de orden cero (Tabla 2).

Tabla 2. Regresiones lineales simples suponiendo modelos cinéticos de orden cero, primer orden y segundo orden para la primera fase y la segunda fase

\begin{tabular}{lccc}
\hline \multicolumn{1}{c}{ Aislado bacteriano } & $\begin{array}{c}\text { Ecuación ajustada } \\
\text { Primera Fase }\end{array}$ & $\mathbf{R}^{\mathbf{2}}$ & Orden \\
& {$[\mathrm{Cr}]=-1,15675 \mathrm{t}+59,099$} & $-0,970767$ & 0 \\
\hline Bacillus thuringiensis & {$[\mathrm{Cr}]=-1,11142 \mathrm{t}+55,878$} & $-0,956407$ & 0 \\
Bacillus amyloliquefaciens & {$[\mathrm{Cr}]=-1,07508 \mathrm{t}+55,294$} & $-0,956642$ & 0 \\
Paenibacillus sp & Segunda Fase & & \\
& {$[\mathrm{Cr}]=0,000021871 \mathrm{t}+0,00486532$} & 0,967029 & 0 \\
Bacillus thuringiensis & $\mathrm{Ln}[\mathrm{Cr}]=-0,00225488 \mathrm{t}+2,76459$ & $-0,986595$ & 1 \\
Bacillus amyloliquefaciens & $1 /[\mathrm{Cr}]=0,000144885 \mathrm{t}+0,0628138$ & 0,977958 & 2 \\
Paenibacillus sp & & & \\
\hline
\end{tabular}

Teniendo en cuenta el comportamiento de los datos se aplicó un análisis de varianza simple (ANOVA), con el fin de determinar si existen diferencias significativas en el porcentaje de reducción entre las tres bacterias y de estas respecto al control; sin embargo, los resultados indicaron que no existen diferencias estadísticamente significativas en la eficiencia de reducción de Cromo entre las bacterias $(\mathrm{p}=$ $0,9953 \alpha=0,05$ ). Adicionalmente, se determinó que hay diferencias significativas en la reducción de Cromo de las bacterias respecto al control ( $p$ $=0 \alpha=0,05$ ).

Para realizar estudios sobre la influencia de la presencia del inóculo bacteriano en una muestra de agua de Río Pasto, se seleccionó la especie bacteriana $B$. thuringiensis dado que fue el aislamiento que presentó mayor porcentaje de reducción.

\section{Evaluación de la eficiencia en la reducción de Cr (VI) por Bacillus thuringiensis en un tratamiento tipo Batch utilizando como sustrato agua residual municipal}

Se evaluó la reducción de $\mathrm{Cr}$ (VI) por $B$. thuringiensis en tres diferentes tratamientos: 1 ) agua sin esterilizar del Río Pasto (Tratamiento $1), 2$ ) agua estéril del Río Pasto inoculada con $B$. thuringiensis (Tratamiento 2), y 3) agua $\sin$ esterilizar del Río Pasto inoculada con $B$. thuringiensis) (Tratamiento 3). Se obtuvo que el Cromo (VI) fue reducido en 74,46\%; 76,12\% y $99,42 \%$ en el proceso en el tratamiento 1 , tratamiento 2 y tratamiento 3 , respectivamente. En la Figura 5 se observa la cinética de reducción de Cromo hexavalente para los tres tratamientos, donde se presenta una fase inicial rápida en el intervalo de 0 a $36 \mathrm{~h}$, transcurrido este tiempo, los tratamientos uno y dos presentan una segunda fase semi-constante en el intervalo de 36 a 156 h; sin embargo, en el tratamiento tres se distingue la segunda fase semi-constante en el intervalo de 36 a $72 \mathrm{~h}$, seguido de una última fase de reducción más acentuada en el intervalo de 72 a $156 \mathrm{~h}$.

Con relación a los datos obtenidos, se determinó que existen diferencias significativas $(p=0 \alpha=$ 0,05 ) entre los tratamientos; adicionalmente, la prueba Tukey permitió identificar que el tercer tratamiento presenta diferencias respecto a los demás tratamientos.

Al analizar los datos de concentración de Cromo hexavalente y tiempo de cada aislado por mínimos cuadrados para cada fase de 36 a $156 \mathrm{~h}$ (tratamiento uno y dos) y de 72 a $156 \mathrm{~h}$ (Tratamiento tres). La Tabla 3 muestra las regresiones lineales y los respectivos valores del 
coeficiente de correlación y las ecuaciones de datos de concentración de Cromo hexavalente para los tres tratamientos.

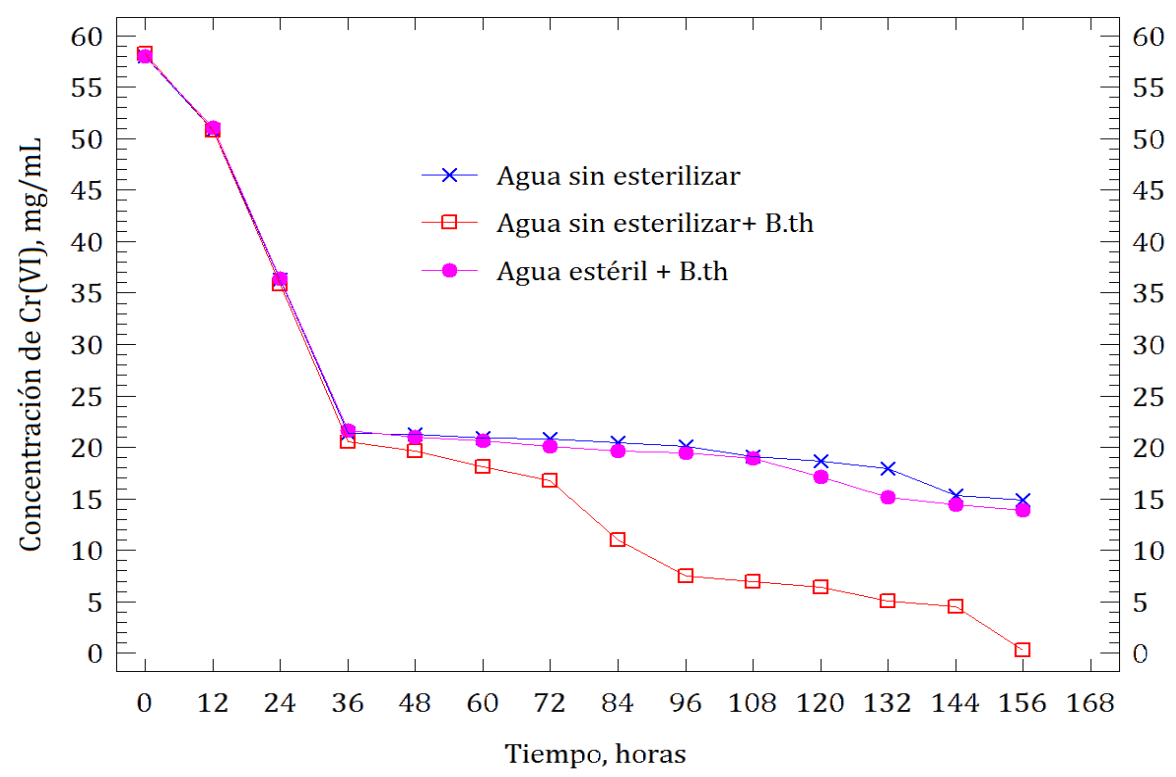

Figura 5. Cinética de la reducción de Cromo hexavalente de Bacillus thuringiensis en tres diferentes tratamientos

Tabla 3. Regresiones Lineales para cinética de reacción de Bacillus thuringiensis en la segunda fase

\begin{tabular}{|c|c|c|c|}
\hline Aislado bacteriano & Ecuación ajustada & $\mathbf{R}^{2}$ & Orden \\
\hline Agua sin esterilizar Río Pasto (36-156h) & {$[\mathrm{Cr}]=-0,0473535 \mathrm{t}+23,8438$} & $-0,913249$ & 0 \\
\hline $\begin{array}{l}\text { Agua estéril Río Pasto + B. thuringiensis } \\
(36-156 \mathrm{~h})\end{array}$ & {$[\mathrm{Cr}]=-0,0645354 \mathrm{t}+24,6202$} & $-0,949390$ & 0 \\
\hline $\begin{array}{l}\text { Agua sin esterilizar Río Pasto }+B \text {. } \\
\text { thuringiensis }(72-156 \mathrm{~h})\end{array}$ & $1 /[\mathrm{Cr}]=0,00211741 \mathrm{t}-0,08 \mathrm{da} 60972$ & 0,988350 & 2 \\
\hline
\end{tabular}

Los datos se ajustan más a cinéticas de orden cero para los tratamientos uno y dos, y para el tratamiento tres se ajustan mejor a una cinética de segundo orden respecto a la concentración de Cromo, mostrando así que la cinética de reducción de Cromo hexavalente realizada por $B$. thuringiensis usando agua sin esterilizar Río Pasto, no solo depende de la reducción de Cromo hexavalente realizada por $B$. thuringiensis.

\section{Discusión}

La reducción de Cromo hexavalente es analiza por cada aislado bacteriano Bacillus thuringiensis, B. amyloliquefaciens, y Paenibacillus $s p$, mostró buenos porcentajes de reducción $83,05 \%, \quad 80,95 \%$ y 79,52\%, individualmente. Es importante señalar que el tratamiento control por sí mismo, no representó ser un medio de reducción de Cromo hexavalente. Los datos experimentales fueron evaluados aplicando regresión lineal simple y 
suponiendo modelos cinéticos de orden cero, primer orden y segundo orden. Cabe mencionar, que los procesos de reducción de Cromo en sistemas biológicos son cinéticas muy complejas, que son influenciadas por diversos factores $(\mathrm{pH}$, temperatura, concentración, etc), por lo cual, se pretende analizar un comportamiento general de acuerdo a la información experimental.

Analizando los dos comportamientos en la reducción de la concentración de Cromo (VI) en los intervalos de tiempo de $0-36$ horas y $36-$ 156 horas, existe una fase inicial rápida $(0-36$ horas), para los tres aislados bacterianos, donde los porcentajes de reducción de $\mathrm{Cr}(\mathrm{VI})$ fueron de $76,15 \% ; 75,46 \%$ y $73,60 \%$ para $B$. thuringiensis, B. amyloliquefaciens, y Paenibacillus $s p$, respectivamente; aplicando mínimos cuadrados a los datos de concentración de Cromo hexavalente y tiempo para cada aislado (Tabla 3) se puede proponer que la cinética de reducción es de orden cero, mostrando que la cinética de reducción no depende de la concentración de Cr(VI); este orden es característico de procesos heterogéneos donde la velocidad es independiente de la concentración de los reactivos ${ }^{23,24}$, se podría suponer que esta fase implica la adsorción física o intercambio iónico en la célula superficie ${ }^{25}$. Este fenómeno también puede estar asociado al crecimiento de la bacteria, ya que como se evidencia en estudios similares, en la fase de crecimiento exponencial existe un aumento en la velocidad de reducción ligada a procesos metabólicos que generan un incremento de la población bacteriana ${ }^{7}$.

Por otro lado, analizando la segunda fase lenta (36 - 156 horas), para los tres aislados bacterianos los porcentajes de reducción de Cr(VI) fueron de 20,14\%; 30,91\% y 22,45\% para B. thuringiensis, B. amyloliquefaciens, y Paenibacillus sp, respectivamente; aplicando mínimos cuadrados a los datos de concentración de Cromo hexavalente y tiempo para cada aislado (Tabla 3) sugiriendo que la cinética de reducción es de orden cero, orden uno y orden dos, respecto a la concentración de Cromo para B. thuringiensis, B. amyloliquefaciens, y Paenibacillus sp, comparativamente.
En este comportamiento semi-constante, se puede inferir que bajo condiciones de estrés inducido las bacterias experimentan una etapa constante de crecimiento, donde el proceso de reducción puede estar asociado a mecanismos de absorción-expulsión, de tal forma que el microorganismo invierte gran cantidad de energía en el proceso de transporte activo para controlar la entrada masiva del catión $\mathrm{Cr}(\mathrm{VI})$ altamente tóxico, frenándose considerablemente los procesos de división celular, al punto que la población viable permanece en un estado de equilibrio ${ }^{26}$.

Para realizar estudios sobre la influencia de la presencia del inóculo bacteriano en una muestra de agua de Río Pasto, se seleccionó la especie bacteriana $B$. thuringiensis dado que fue el aislamiento bacteriano que presentó mayor porcentaje de reducción; esto se debe probablemente a que la bacteria presenta un metabolismo anaerobio facultativo $y$ quimiorganotrofo, características que facilitan la adaptación a las condiciones del proceso de fermentación y favorecen el mecanismo de reducción ${ }^{27}$. En cuanto al tratamiento control empleado para este proceso (Figura 3) se observó que la concentración de Cr (VI) inicial, no varió significativamente en todo el proceso, indicando de cierta manera, que las bacterias empleadas en el tratamiento son las responsables de la reducción de este metal; aparentemente, ningún otro compuesto del medio o sustrato empleado actúa como agente reductor.

Debido a la capacidad metabólica de $B$. thuringiensis para realizar la reducción de $\mathrm{Cr}(\mathrm{VI})$ a $\mathrm{Cr}(\mathrm{III})$, hay un interés creciente en esta bacteria en procesos de biorremedación; estudios indican que $B$. thuringiensis aislado de agua residual industrial presentó un porcentaje de reducción del $82 \%$ en un medio sintético (Luria Bertani) a $37{ }^{\circ} \mathrm{C}$ y $48 \mathrm{~h}^{28}$. Igualmente, B. thuringiensis aislado de la rizósfera de coliflor en un cultivo irrigado con agua de efluentes industriales, mostró una absorción del $80 \%$ de $\operatorname{Cr}(\mathrm{VI})$; esta absorción posiblemente se debe a la presencia de grupos funcionales (amino, carboxilo, hidroxilo, 
carbonilo) en la superficie de la membrana que facilitan la interacción con el metal ${ }^{29}$.

En cuanto a Paenibacillus sp., varios autores describen que bacterias pertenecientes a este género como, $P$. xylaniliticus presentan la capacidad de reducir aproximadamente el 80,43\% de Cromo, en una concentración inicial de 19,7 mg/L después de $72 \mathrm{~h}^{30}$. Tiwari et al., evidenciaron la reducción de $\mathrm{Cr}$ (VI) de $P$. macerans, con una eficiencia del $99 \%$ a $35{ }^{\circ} \mathrm{C}$ y $\mathrm{pH} 8,0^{31}$. Al respecto, se reporta que el género Paenibacillus tiene la capacidad de secretar polímeros extracelulares como, polisacáridos que incrementan la afinidad con metales pesados, un caso relacionado fue descrito por Shiomi ${ }^{32}$ en el que $P$. jamilae presenta un aumento en la afinidad por Plomo atribuida a los polisacáridos que secreta. Por otra parte, Rath, et al., indican que $B$. amyloliquefaciens aislada de una mina de cromita presenta la capacidad de producir una enzima extracelular (Cromato reductasa) que permite la reducción de Cromo hexavalente a Cromo trivalente cuando se encuentra en condiciones de estrés; por tal motivo se infiere que esta bacteria presenta un gran potencial en cuanto a procesos de biorremediación en ambientes contaminados con metales pesados ${ }^{32,33}$. Paralelamente, Das et al., reportan que $B$. amyloliquefaciens aislado de suelo de una mina de cromita en la India presentó alta tolerancia a Cr (VI) (900 mg/L) bajo condiciones óptimas, $\mathrm{pH} 7 \mathrm{y}$ temperatura de $35^{\circ} \mathrm{C}^{34}$.

Por lo anteriormente expuesto, se infiere que las bacterias silvestres aisladas de ambientes contaminados con este metal, pueden ser aplicadas en procesos de biorremediación, gracias a su amplia diversidad metabólica y funcional. Las especies bacterianas fuente de este estudio tienen la capacidad de habitar y regular su densidad poblacional en ambientes contaminados con Cromo hexavalente, esto posiblemente se debe a que la acumulación gradual de cationes de Cromo y su alta toxicidad tanto en lugares intervenidos por el hombre, como en ambientes naturales; estas bacterias; han generado durante la evolución, procesos de adaptación espontánea; por medio del desarrollo o adquisición de sistemas genéticos que ayudan a contrarrestar el efecto de elevadas concentraciones de Cromo, considerando que el desarrollo de una población resistente a metales en entornos contaminados puede ser resultado de transferencia vertical de genes (la reproducción), transferencia horizontal de genes (incluyendo transposones y plásmidos con amplia gama de hospederos), y presiones de selección en mutantes espontáneos (debido a la presencia de metales) ${ }^{35}$.

Microorganismos pertenecientes a los géneros Bacillus y Paenibacillus, presentan mecanismos de reducción enzimática asociados a enzimas membranales o de la fracción soluble que intervienen en la reducción del Cromo hexavalente, entre estas enzimas se encuentra la ChrR la cual muestra una actividad reductora dependiente del NADH en condiciones aerobias, durante la reducción de $\mathrm{Cr}(\mathrm{VI})$ la proteína ChrR muestra actividad de quinona reductasa generando una flavínsemiquinona; gracias a esta reacción la enzima transfiere alrededor del 25\% de los electrones del NADH al anión superóxido, de modo que en una ruta la enzima reduce el $\mathrm{Cr}(\mathrm{VI})$ a $\mathrm{Cr}(\mathrm{III})$, generando $\mathrm{Cr}(\mathrm{V})$ como intermediario y el anión superóxido, y por un mecanismo adicional reduce las quinonas, lo cual provee de un mecanismo de protección contra las especies reactivas de oxígeno. Teniendo en cuenta lo anterior, se puede deducir que las bacterias empleadas en este estudio pueden estar utilizando un mecanismo similar, pues el proceso de fermentación se llevó acabo en condiciones aerobias ${ }^{33,36}$.

B. thuringiensis fue el aislado bacteriano que presentó mejor reducción de Cromo hexavalente, se consideró esta bacteria en tres diferentes tratamientos (1) agua sin esterilizar del río Pasto, (2) agua estéril del río Pasto inoculada con $B$. thuringiensis y (3) agua sin esterilizar del río Pasto inoculada con $B$. thuringiensis) y se obtuvo porcentajes de reducción de 74,46\%; 76,12\% y 99,42\% en el proceso de reducción de Cr (VI), para cada tratamiento.

La diferencia entre el comportamiento de los dos primeros tratamientos respecto al tercero, 
probablemente se debe a la interacción de $B$. thuringiensis con microorganismos presentes en el agua, dado que la presencia de asociaciones entre microorganismos confiere ventajas a la adaptación frente a ambientes adversos; adicionalmente, Gómez de León et al., en el año 2015 describen que la capacidad de adhesión de las esporas de $B$. thuringiensis, facilita la formación de biopelículas y proporciona el sustrato necesario para la interacción entre diferentes microorganismos ${ }^{37}$.

El tratamiento con mayor porcentaje de reducción de Cromo hexavalente, en un periodo de $156 \mathrm{~h}$, fue el tratamiento tres $(99,42 \%)$ debido a que, las condiciones establecidas dentro del biorreactor probablemente favorecieron el establecimiento de asociaciones entre $B$. thuringiensis y organismos afines presentes en el agua. Observaciones similares fueron reportadas por Muneer et al., quienes evaluaron individualmente y en combinaciones diferentes, la reducción de $\mathrm{Cr}(\mathrm{VI})$ de tres microorganismos (B. thuringiensis, Candida etschellsii y Stylonychiamytilus) aislados de aguas residuales industriales, con un porcentaje de reducción del $82 \%, 80 \%$ y $60 \%$ para bacteria, levadura y protozoo, respectivamente. La combinación de los tres organismos presentó un porcentaje de reducción de $90 \%$ a las 96 h, concluyendo, que este tipo de consorcios o asociaciones favorece la reducción de este metal ${ }^{28}$.

Recientemente, se estimó el porcentaje de reducción de un consorcio microbiano conformado en su mayoría por bacterias del genero Bacillus, el proceso se observó aproximadamente durante siete semanas, trascurrido este tiempo, las únicas especies bacterianas que persistieron fueron, Enterobacter sp., B. cereus y B. thuringiensis, demostrando la gran capacidad de adaptación al medio de las bacterias y su eficiencia en la reducción del metal $(100 \%)^{38}$.

En consecuencia, se infiere que B. thuringiensis al igual que otras especies del género Bacillus presentan, características metabólicas favorables para su implementación en sistemas de biorremediación, como lo reportan varios estudios, la eficiencia en el proceso de transformación de Cr (VI) a Cr (III) de la bacteria es significativamente alta, tanto individualmente como en consorcio en relación a otros microorganismos ${ }^{29,39}$. En este orden de ideas, estudios independientes de Molokwane et al., determinaron que al emplear un cultivo mixto de bacterias, la reducción biocatalítica del $\mathrm{Cr}$ (VI) tiene mayor eficiencia, condicionalmente ninguno de los cultivos individuales alcanzó el mismo nivel de reducción de Cromo. Finalmente, mediante la caracterización de los microrganismos se encontró que el género Bacillus predominó en estos cultivos ${ }^{30}$.

En relación a lo antes descrito, Panneerselvam et al., aislaron seis bacterias de suelo contaminado con desechos de curtiembres y valoraron el potencial de reducción de cada bacteria y en consorcio, concluyendo que el consorcio es más eficiente en la reducción de Cromo hexavalente comparado con cada bacteria; y mediante la caracterización de las cepas se determinó que pertenecían a las especies, Bacillus endophyticus, Microbacterium paraoxydans y Bacillus simplex; de tal forma que lo citado sugiere la existencia de asociaciones que optimizan la eficiencia de mecanismos que posiblemente les permite a las bacterias tolerar o reducir el Cromo hexavalente ${ }^{39,40 .}$.

Secuencialmente, otros autores demostraron que algunos microorganismos pierden viabilidad en presencia de altas concentraciones de Cr (VI); no obstante, al encontrarse en cultivos mixtos, estos microorganismos logran tolerar y reducir el metal en concentraciones de 100 a $300 \mathrm{mg} / \mathrm{L}$, utilizando diferentes donadores de electrones; por tal motivo es esencial tener en cuenta la importancia de la formación de asociaciones entre microorganismos, cuando estos se encuentran en ambientes desfavorables ${ }^{41}$.

\section{Conclusiones}

Los aislados bacterianos $B$. thuringiensis, $B$. amyloliquefaciens, y Paenibacillus $\underline{s p}$. presentan la capacidad de tolerar concentraciones de 59 $\mathrm{mg} / \mathrm{L}$ de Cromo hexavalente y alcanzan porcentajes de reducción entre el 79 y $83 \%$, en 
biorreactores con agua estéril residual municipal.

La bacteria $B$. thuringiensis presentó alta eficiencia en la reducción de Cromo hexavalente $(99,42 \%)$ cuando fue implementada en un tratamiento a escala de laboratorio de agua residual sin esterilizar, comparada con técnicas fisicoquímicas convencionales no tan efectivas para la reducción del metal.

\section{Recomendaciones}

Evaluar la reducción de Cromo hexavalente de las bacterias B. amyloliquefaciens y Paenibacillus $s p$., implementando como sustrato agua residual sin esterilizar del río Pasto.

Evaluar la reducción de Cromo hexavalente a trivalente de un cultivo mixto de las tres bacterias (B. thuringiensis, B. amyloliquefaciens y Paenibacillus sp.).

Optimizar el proceso de reducción de Cromo hexavalente y cuantificar la cantidad de Cromo total, a partir de aguas residuales.

Implementar técnicas que permitan conocer la composición de la comunidad microbiana presente en el tratamiento.

Estimar relaciones cinéticas de la reducción de Cromo hexavalente realizado por las bacterias y evaluar la influencia del pH en la reducción de $\mathrm{Cr}$ (VI).

\section{Financiación}

La presente investigación fue realizada con recursos propios de los grupos de investigación de Biotecnología Microbiana y de investigación en Biología Matemática y Matemática Aplicada GIBIMMA, de la Universidad de Nariño.

\section{Agradecimientos}

Los autores expresan sus agradecimientos al personal técnico de laboratorios especializados de la Universidad de Nariño y al proyecto "Fortalecimiento de capacidades regionales en investigación, desarrollo tecnológico e innovación en el Departamento de Nariño", Fondo de CTeI del Sistema General de Regalías desarrollado por la Fundación CEIBA en convenio con la Gobernación de Nariño.

Conflicto de intereses: Ninguno declarado.

\section{Referencias}

1. Guevara D. Biorremoción de Cromo (Cromo total y Cromo VI) en agua sintética por dos inóculos bacterianos nativos compuestos, a escala de laboratorio. Trabajo de grado previo a la obtención de título de Ingeniera en Biotecnología. Sangolquí: Escuela Politécnica del Ejército., Ecuador; 2010.

2. Caballero G, Silva J, Cervantes C, Ramírez M. Distribución de los genes chrA en bacterias de origen nosocomial. Ciencia Nicolaita. 2012; 56(2012):79-90.

3. Bakiyaraj R, Baskaran L, Chidambaram A, Mahakavi T, Santhoshkumar M. Bioremediation of Chromium by Bacillus subtilis and Pseudomonas aeruginosa. Curr. Microbiol. App. Sci. 2014; 3(9):715-719.

4. Agencia para Sustancias Tóxicas y el Registro de Enfermedades. ToxFAQs ${ }^{\mathrm{TM}}$ - Cromo. [Online].; 2012 [cited 2016 mayo 5. Disponible en: http://www.atsdr.cdc.gov/es/toxfaqs/es_tfacts7.html

5. Arango C, Alzate AM. Proyecto gestión ambiental en la industria de curtiembre en Colombia. Colombia; 2004.

6. Benítez-Campo N. Producción Limpia Y Biorremediación Para Disminución De La Contaminación Por Cromo En La Industria De Curtiembres. Ambiente y Sostenibilidad. 2011 Febrero; 1(1):25-31.

7. Ramírez A, Benítez-Campo N. Tolerancia y reducción de Cromo (VI) por Bacillus cereus b1, aislado de aguas residuales de una curtiembre. Revista de ciencias. 2013 diciembre; 17(2):51-63.

8. Gutiérrez JF, Espino AE, Coreño A, Acevedo FJ, Reyna GE, Fernández FJ, et al. Mecanismos de intracción con Cromo y aplicaciones biotecnológicas en hongos. Rev latinoam Biotecnol Amb Algal. 2010; 1(1):47-63.

9. Rengifo-Gallego AL, Peña-Salamanca E, Benítez-Campo N. Efecto de la asociación alga-bacteria Bostrychia calliptera (Rhodomelaceae) en el porcentaje de remoción de Cromo en laboratorio. Rev. Biol. Trop. 2012 Marzo; 60(3):1055-1064.

10. Williams PJ, Botes E, Maleke MM, Ojo A, DeFlaun M, Howell J, et al. Effective bioreduction of hexavalent chromium-contaminated water in fixed-film bioreactors. Water SA. 2014 July; 40(3):549-554.

11. Otero ID. Remoción de la materia orgánica de las aguas residuales vertidas al Río Pasto con bacterias productoras de polihidroxialcanoatos. Trabajo de grado presentado como requisito parcial para optar al título de Biólogo. Pasto: Universidad de Nariño, Colombia; 2012.

12. Guerrero DL, Pinta J, Otero-Ramirez ID, Fernandez P. Reducción de Cromo por bacterias productoras de polohidroxialcanoatos. Pasto: Universidad de Nariño, Colombia; 2012.

13. Guerrero DL, Pinta J, Burbano-Rosero EM, Fernández P. Densidad poblacional de Bacillus amyloliquefaciens, B. 
thuringiensis y Paenibacillus sp., sometidas In Vitro a diferentes concentraciones de Cromo hexavalente. Pasto: Universidad de Nariño, Colombia; 2014.

14. Parra LM. Operación de un filtro anaerobio de flujo ascendente (FAFA) hasta alcanzar el estado estable. Trabajo de Grado para optar al titulo de Ingeniera Química. Manizales: Universidad Nacional de Colombia, Colombia; 2006.

15. Gunjate JK, Gholse SB, Khope RU. A simulation study of the removal efficiency of modified granular activated carbon on Cobalt. International Journal of Chemical and Physical Sciences. 2014 March; 3(2):29-34.

16. American Public Health; Association, American Water Works; Federation, Water Environment. Chromiun 117A Hexavalente chromiun. In Health AP, Association AWW, Federation WE. Standards Methods for the examination of water and wastewater.; 1999. p. 271.

17. Erazo R, Cárdenas J. Determinación experimental del coeficiente de transferencia de oxígeno (kLa) en un biorreactor batch. Peruana de Química e Ingeniería Química. 2001; 4(2):22-27.

18. Martínez M. Aislamiento de cepas nativas bacterianas a partir de biopelícula obtenida de un sitio de vertimiento de aguas residuales con alto contenido de Cromo. Tesis Magíster en Biotecnología. Medellín: Universidad Nacional de Colombia, Antioquia; 2009.

19. Ronald T, Lester C. The Chromium-Diphenylcarbazide Reaction 1. Anal. Chem. 1956; 78(19):4862-4866.

20. Bremer H, Fessel C, PezzatoIII L, Padovanil C. The spectrophotometric method on the routine of 1,5diphenylcarbazide was adjusted on chromium determination in feces, after its utilization as a biological marker as chromium (III) oxide. Cienc. Rura. 2005 may; 35(3):691-697.

21. Herrera G, Ordoñez P, Anaguano A. Estandarización de la difenilcarbazida como indicador y acomplejante en la identificación de Cromo hexavalente-Cr (VI). Producción+ Limpia. 2013 Julio;8(2):9-20.

22. Skoog DA, West DM, J HF, Crouch SR. Fundamentos de Química Analítica. Octava ed. Barcelona: Reverte; 2005.

23. Levine IN. Fisicoquímica. Quinta ed. Madrid: McGrawHill; 2004.

24. Srinath T VTRPGS. Chromium (VI) biosorption and bioaccumulation by chromate resistant bacteria. Chemosphere. 2002 September; 48(4):427-435.

25. Ahemad M. Implications of bacterial resistance against heavy metals in bioremediation: a review. IIOABJ. 2012 June; 3(3):39-46.

26. Geets J, Vangronsveld J, Diels L, Taghavi S, Van der Lelie D. Microbial activities, monitoring and application as part of a management strategy for heavy metalcontaminated soil and ground water. Developments in Soil Science. 2008 March; 32(2008):521-559.

27. Sauka D, Benintende G. Bacillus thuringiensis: generalidades. Un acercamiento a su empleo en el biocontrol de insectos lepidópteros que son plagas agrícolas. Revista Argentina de Microbiología. 2008 Marzo; 40(2):124-140.

28. Muneer B, Rehman A, Shakoori F, Shakoor A. Evaluation of consortia of microorganisms for efficient removal of hexavalent chromium from industrial wastewater. Bulletin of environmental contamination and toxicology. 2009 January; 82(5):597-600.

29. Oves M, Khan M, Zaidi A. Biosorption of heavy metals by Bacillus thuringiensis strain OSM29 originating from industrial effluent contaminated north Indian soil. Saudi journal of biological sciences. 2013 April; 20(2):121-129.

30. Molokwane PE, Meli KC, Nkhalambayausi-Chirwa EM. Chromium (VI) reduction in activated sludge bacteria exposed to high chromium loading: Brits culture (South Africa). Water Res. 2008 August; 42(17):4538-4548.

31. Tiwari S, Singh SN, Garg SK. Bacterial Reduction of $\mathrm{Cr}$ (VI) and Fe (III) in In Vitro Conditions. Bioremediation Journal. 2014 April; 18(2):158-168.

32. Shiomi N. An assessment of the Causes of Lead Pollution and the Efficiency of Bioremediation by Plants and Microorganisms. In Shiomi N, editor. Advances in Bioremediation of Wastewater and Polluted Soil.: InTech; 2015. p. 247-274.

33. Rath B, Das S, Mohapatra P, Thato H. Optimization of extracellular chromate reductase production by Bacillus amyloliquefaciens (CSB 9) isolated from chromite mine environment. Biocatalysis and Agricultural Biotechnology. 2014 July; 3(3):35-41.

34. Das S, Pradhan SK, Sudarshan M, Chakraborty A, Thatoi HN, Pandey SK. Genomic Analysis and Comparative Hexavalent Chromium Reduction Potential of Predominant Bacillus species Isolated from Chromite Mine Soil. Soil. Sediment. Contam. 2015; 24(2): 206221.

35. Camacho A, Fernández-Valiente E. Un mundo dominado por los microorganismos. Ecología microbiana de los lagos antárticos. Revista Ecosistemas. 2005 Mayo; 14(2):66-78.

36. Gutiérrez JF, Cervantes C. Interacciones microbianas con el Cromo: mecanismos y potencial biotecnológico. CONCYTEG. 2008 Julio; 3(37):21-36.

37. Gómez de León P, Ibarra JE, Bravo A, García-Gutiérrez K. Adhesión de la capacidad de Bacillus thuringiensis esporas y su relación con la formación de biopelículas. SOJ Microbiol Infect Dis. 2015; 3(2):1-6.

38. Mtimunye JP, Chirwa EM. Finite difference simulation of biological chromium (VI) reduction in aquifer media columns. Water Research Commission. 2014 April; $40(2): 359-368$.

39. Joutey NT, Bahafid W, Sayel H, Ananou S, El Ghachtouli N. Hexavalent chromium removal by a novel Serratia proteamaculans isolated from the bank of Sebou River (Morocco). Environ. Sci. Pollut. R. 2014 February ; 21(4):3060-3072.

40. Panneerselvam P, Choppala G, Kunhikrishnan A, Bolan N. Potential of novel bacterial consortium for the remediation of chromium contamination. Water Air Soil Pollut. 2013 November; 224(12):1-11.

41. Coelho LM, Rezende HC, Coelho LM, Sousa PA, Melo DF, Coelho NM. Bioremediation of Polluted Waters Using Microorganisms. In Shiomi N, editor. Advances in Bioremediation of Wastewater and Polluted Soil.: InTech; 2015. p. 1-22. 